\title{
Esophageal recanalization by combined antegrade/retrograde esophagoscopy for radio- therapy-induced complete esophageal occlusion
}

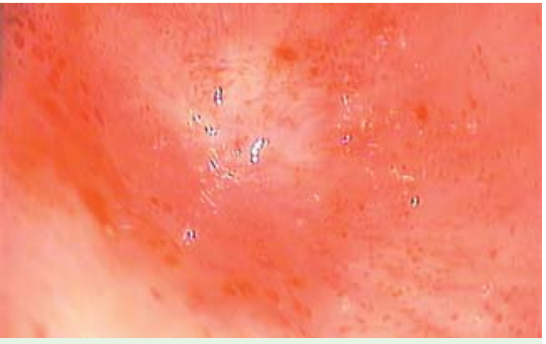

Fig. 1 Antegrade view of the upper esophagus showing complete occlusion of the esophageal lumen.

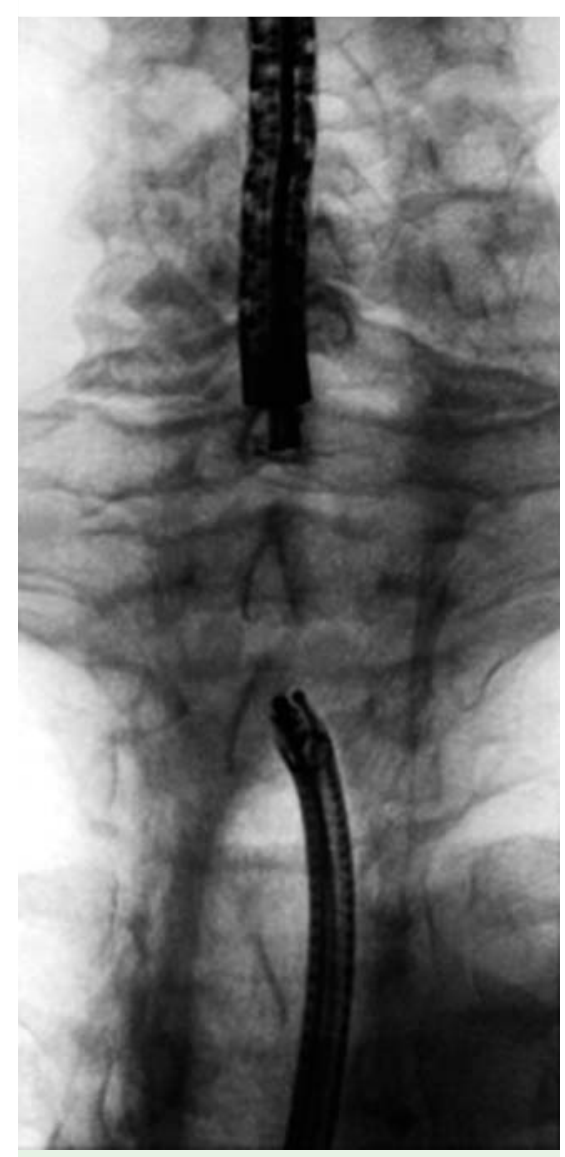

Fig. 2 Radiographic view of the occlusion during combined antegrade/retrograde esophagoscopy showing that the length of the occlusion was about $40 \mathrm{~mm}$.

One side effect of radiochemotherapy of head and neck malignancies is the development of esophageal stenosis [1,2]. Subsequent therapeutic options include endoscopic balloon dilation or bouginage, with the option to perform combined ante-
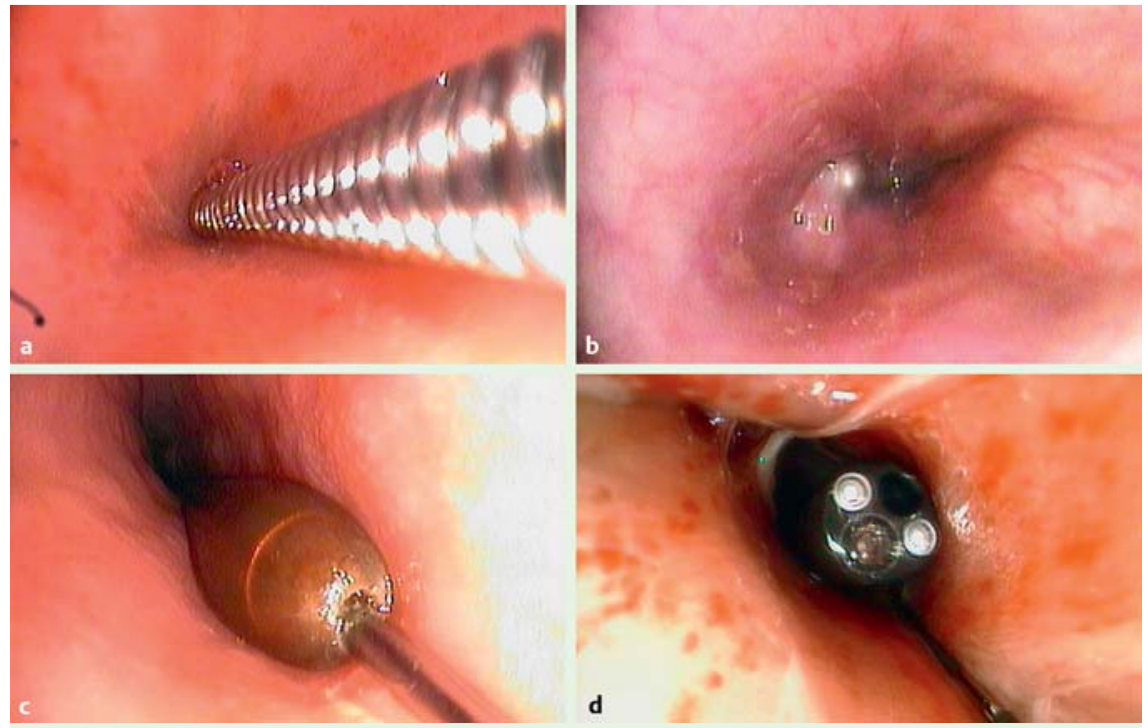

Fig. 3 Endoscopic views showing recanalization of the esophageal lumen. a A wire is passed into the occluded esophagus by antegrade puncture; $\mathbf{b}$ under direct visualization via the retrograde endoscope; c wire-guided bouginage is then performed; and finally $\mathbf{d}$ an endoscope is passed through the fully recanalized lumen.
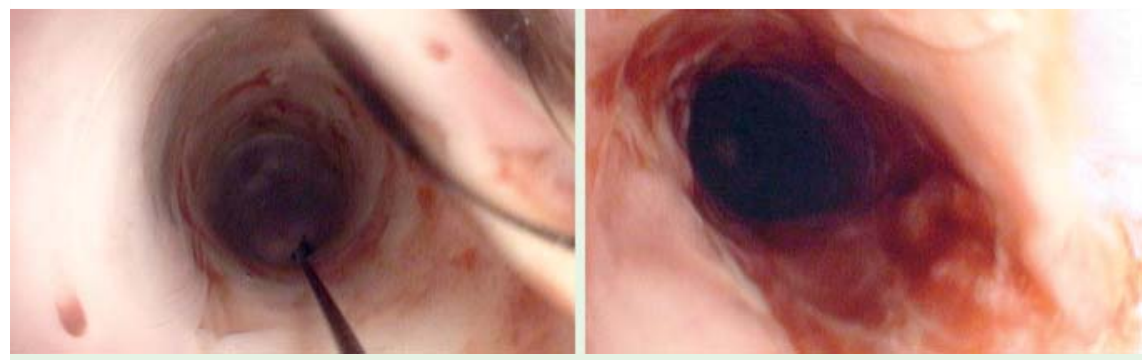

Fig.4 Standard endoscopic balloon dilation of the reopened lumen showing a good final result.

grade/retrograde esophagoscopy in cases of complete esophageal occlusion [3-8], or major surgery, such as esophageal resection, with its high risk of surgical complications [9]. The limitations of endoscopic therapy for complete esophageal closure have been defined and include long-segmental occlusions over $30 \mathrm{~mm}$ in length, in which case it is recommended to achieve patency of the neo-esophagus using stents, although there is a risk of stent-associated complications [5-8].

This report describes an endoscopic recanalization using a rendezvous maneuver in a 54-year-old patient with complete occlusion of the upper esophagus after primary radiochemotherapy for a hypopharyngeal squamous cell carcinoma.
Enteral feeding had been provided via a percutaneous endoscopic gastrostomy (PEG). The patient reported grade IV dysphagia 6 weeks after the radiochemotherapy, which did not resolve with logopedic training. He was seen in our endoscopy unit 12 months later for endoscopic therapy of his suspected upper esophageal stenosis.

Endoscopic examination showed complete occlusion of the upper esophagus $(\bullet$ Fig. 1). Simultaneous transoral and retrograde esophagoscopy (via the PEG channel) with fluoroscopy revealed a long-segmental occlusion of about $40 \mathrm{~mm}$ in length ( $\bullet$ Fig.2). Recanalization was performed by perforating the occlusion with an Eder-Puestow wire passed from 
the oral direction under direct visualization with the retrograde trans-PEG endoscope. Following this, wire-guided bouginage with Savary-Miller bougies up to a size of $12 \mathrm{~mm}$ was possible ( $\bullet$ Fig.3). Patency of the neo-esophagus was ensured by simply placing a nasogastric tube. The complete procedure lasted 43 minutes and the patient was discharged from hospital after 3 days without the nasogastric tube, having had no post-intervention complications.

In the following weeks, the patient underwent recurrent balloon dilations, up to a size of $18 \mathrm{~mm}$ ( Fig. 4 ), which was combined with logopedic training. Eventually, 2 years after radiochemotherapy, the patient is now free of cancer, able to swallow almost normally, and it has finally been possible to remove his PEG.

In conclusion, recanalization of a complete esophageal occlusion by rendezvous esophagoscopy is possible even in longsegmental esophageal occlusions and without the use of esophageal stents to achieve patency.

Endoscopy_UCTN_Code_TTT_1AO_2AH

Competing interests: None
Markus Alexander Küper ${ }^{1}$, Dietmar Stüker², Alfred Königsrainer², Thomas Kratt ${ }^{2}$

${ }^{1}$ Department for General and Visceral Surgery, Bundeswehr Hospital Berlin, Germany

${ }^{2}$ Department for General, Visceral and Transplant Surgery, University Hospital Tübingen, Germany

\section{References}

1 Bueno R, Swanson SJ, Jaklitsch MT et al. Combined antegrade and retrograde dilation: a new endoscopic technique in the management of complex esophageal obstruction. Gastrointest Endosc 2001; 54: 368-372

2 Laurell G, Kraepelien T, Mavroidis $P$ et al. Stricture of the proximal esophagus in head and neck carcinoma patients after radiotherapy. Cancer 2003; 97: 1693-1700

3 Pereira-Lima JC, Ramires RP, Zamin I Jr et al. Endoscopic dilation of benign esophageal strictures: report on 1043 procedures. Am J Gastroenterol 1999; 94: 1497-1501

4 Van Twisk J, Brummer RJM, Manni J. Retrograde approach to pharyngo-esophageal obstruction. Gastrointest Endosc 1998; 48: 296-299

5 Baumgart DC, Veltzke-Schlieker W, Wiedenmann $B$ et al. Successful recanalization of a completely obliterated esophageal stricture by using an endoscopic rendezvous maneuver. Gastrointest Endosc 2005; 61: 473-475

6 Moyer MT, Stack BC Jr, Mathew A. Successful recovery of esophageal patency in 2 patients with complete obstruction by using combined antegrade retrograde dilation procedure, needle knife, and EUS needle. Gastrointest Endosc 2006; 64: 789-792

7 McGrath K, Brazer S. Combined antegrade and retrograde dilation: a new endoscopic technique in the management of complex esophageal obstruction. Gastrointest Endosc 2002; 56: $163-164$

8 Schembre D, Dever JB, Glenn $M$ et al. Esophageal reconstitution by simultaneous antegrade/retrograde endoscopy: re-establishing patency of the completely obstructed esophagus. Endoscopy 2011; 43: 434-437

9 Cho BC, Kim M, Lee JH et al. Pharyngoesophageal reconstruction with a tubed free radial forearm flap. J Reconstr Microsurg 1998; 14 : $535-540$

Bibliography

DOI http://dx.doi.org/ 10.1055/s-0034-1365786

Endoscopy 2014; 46: E510-E511

(c) Georg Thieme Verlag KG

Stuttgart · New York

ISSN 0013-726X

\section{Corresponding author}

\section{Markus Alexander Küper, MD}

Department for General and Visceral Surgery Bundeswehr Hospital Berlin

Scharnhorststraße 13

D-10115 Berlin

Germany

Fax: +49-30-28411043

Mkueper@hotmail.com 